\title{
The Effects of Transdermal Nicotine Therapy for Smoking Cessation on Depressive Symptoms in Patients with Major Depression
}

\author{
Haraldur S. Thorsteinsson, M.A., J. Christian Gillin, M.D., Christi A. Patten, Ph.D., \\ Shahrokh Golshan, Ph.D., Laura D. Sutton, R.N., B.S.N., Sean Drummond, Ph.D., \\ Camellia P. Clark, M.D., John Kelsoe, M.D., and Mark Rapaport, M.D.
}

This study examines the effects of transdermal nicotine patches for smoking cessation on depressive and withdrawal symptoms among 38 non-medicated subjects with Major Depressive Disorder. The study was conducted over a 29day period, which included a 7 day baseline phase, a 14 day treatment phase, and an 8 day placebo phase. During the treatment phase subjects received either active nicotine patches $(N=18)$ or placebo patches $(N=20)$ that were administered in a randomized, double-blind fashion. The target quit date (TQD) was day 8. Significantly, more subjects in the placebo group than in the nicotine group resumed smoking following the TQD (50\% vs. $22 \%)$. There was little evidence for effects of active nicotine patches on measures of mood (HRSD, BDI, POMS) or withdrawal symptoms among subjects that remained abstinent throughout the study $(\mathrm{N}=24)$. Those who resumed smoking had more severe withdrawal symptoms than those who remained abstinent. One patient in the placebo group $(\mathrm{n}=20)$ became more depressed after 2 weeks of abstinence. None of the patients in the nicotine group $(n=18)$ became more depressed.

[Neuropsychopharmacology 24:350-358, 2001] (C) 2001 American College of Neuropsychopharmacology. Published by Elsevier Science Inc.
KEY WORDS: Depressive disorder; Smoking cessation; Nicotine; Withdrawal symptoms; Randomized clinical trial

Psychiatric patients, including patients with schizophrenia, substance abuse disorders, and mood disorders, typically smoke more than the general population

From the San Diego State University and University of California San Diego Joint Doctoral Program in Clinical Psychology, San Diego, CA (HST); the Department of Psychiatry, University of California San Diego, San Diego, CA (HST, JCG, SG, LDS, SD, CPC, JK, MR); Psychiatry Service, Veterans Administration, San Diego Healthcare System, San Diego, CA (JCG, SG, LDS, CPC, JK, MR); and the Department of Psychology and the Nicotine Research Center, Mayo Clinic, Rochester, MN (CAP).

Address correspondence to: J. Christian Gillin, M.D., Psychiatry Service, UCSD/VAMC (116A), 3350 La Jolla Village Dr., San Diego, CA, 92161; Tel.: (858) 534-2137, ext. 3962; Fax: (858)-458-4201; E-mail: jgillin@.ucsd.edu

Received November 16, 1999; revised August 22, 2000; accepted September 14, 2000.
(Hughes et al. 1986). Research over the past 10-15 years indicates that depression is especially associated with smoking. In particular, major depressive disorder (MDD) is more common among smokers than nonsmokers. Glassman et al. (1990) analyzed data from the St. Louis part of the Epidemiological Catchment Area survey and found that the lifetime prevalence of MDD was $10 \%$ among those who had ever smoked compared to $6 \%$ in the general population. Other epidemiological studies have shown similar results (e.g. (Breslau 1995; Kendler et al. 1993), for different age (Breslau et al. 1991; Salive and Blazer 1993) and ethnic (Lee and Markides 1991) subgroups.

Studies have generally shown that individuals who have a history of MDD are less likely to quit smoking and more likely to fail at smoking cessation. This has been observed in cross-sectional epidemiological stud- 
ies (Anda et al. 1990; Breslau et al. 1991; Glassman et al. 1990) and in randomized trials of smoking cessation (Covey et al. 1993; Ginsberg et al. 1995; Glassman et al. $1988,1993)$. The evidence from prospective studies is less clear. One 9 year follow-up study (Anda et al. 1990) reported lower cessation rates among smokers with high depressive symptoms at baseline, while another more recent study that followed 1007 young adults over 5 years reported that history of major depression did not affect rates of cessation (Breslau et al. 1998).

Three types of models have been proposed to account for the association between smoking and depression (see (Borrelli et al. 1996a). The first model, often referred to as the self-medication model, suggests that nicotine has particular reinforcing properties for depressed people who use it to enhance their mood. The second model states that the association is in fact spurious because both depression and smoking are caused by common factors. And the third model states that smoking cessation may lead to depression, particularly in vulnerable people.

There is some evidence for all 3 models. First, nicotine may have antidepressant properties. Salin-Pascual et al. (1995) reported that short-term administration (24 hours up to 4 days) of nicotine patches improved mood in non-smoking depressed patients within $24 \mathrm{hr}$ of administration. Although the antidepressant effects were short lived (reversal of antidepressant effect after 3-4 days) (Salin-Pascual et al. 1996), the effects were quite dramatic (44\% decrease in depression ratings) (SalinPascual and Drucker-Colín 1998). In addition, research has shown a dose-response relationship between nicotine and sensations of euphoria, thus contributing to its antidepressant effects (Pomerleau and Pomerleau 1992). The neurochemical processes underlying the antidepressant effects of smoking remain poorly understood, but there is some evidence that nicotine enhances dopamine release (Lerman et al. 1998) and inhibits monoamine oxidase (Fowler et al. 1996).

Second, some evidence suggests that depression and smoking may be caused by common factors. Kendler et al. (1993) concluded from a study of 1566 twins that "the association between smoking and major depression (MD) arises largely from familial factors, which are probably genetic, that predispose to both smoking and depression" (p. 36). Fergusson et al. (1996) reached similar conclusions in their study of a cohort of 947 16-yearold New Zealand adolescents. They observed that depressive disorder was associated with an increased risk for nicotine dependence, but environmental risk factors common to both nicotine dependence and depression accounted for about half the association. To reconcile the differences between their findings and the findings from Kendler et al., the authors speculated that the risk factors which they identified may describe some of the common pathways by which the genetic predisposition to both smoking and depression may operate.
Finally, smoking cessation may lead to depression, thus contributing to the observed comorbidity. The evidence for this hypothesis comes from several different trials of smoking cessation. Bock et al. (1996) reported three cases of women who developed significant depressive symptoms that required psychiatric intervention, shortly after cessation. Glassman et al. (1993) reported similar findings from 300 subjects in a clinical trial of clonidine for smoking cessation. Out of 126 subjects who successfully completed the trial, nine subjects had an episode of depression within 3 months after cessation (Covey et al. 1997). Furthermore, incidence was dependent on past history of depression and was $2 \%$ in patients with no history, $17 \%$ in those with a history of single episode, and $30 \%$ in those with history of recurrent major depression. Smoking cessation trials with other types of antidepressants have reported similar findings. Borrelli et al. (1996b) reported that 7\% (all with history of MDD) of 114 subjects developed MDD during treatment, and Patten et al. (1999) reported that 5 subjects (4 with a history of MDD) developed severe depression in a trial of buproprion.

Although the evidence reported above is certainly consistent with the hypothesis that smoking cessation may lead to or exacerbate depressive symptoms, it is far from being conclusive. The major limitation of previous studies is the lack of an appropriate control group that allows comparison of rates of new episodes of depression. Another limitation of a more practical nature is that past studies have excluded individuals with current major depression. Since MDD is over-represented in cigarette smokers it is important to understand the effects of different treatments for smoking cessation on depressive symptoms in patients with current depression. This is important since there have not been randomized clinical trials of depressed smokers.

The present study examined the effects of nicotine patch therapy for smoking cessation on depressive and withdrawal symptoms in patients with current MDD. Subjects were randomly assigned to receive either nicotine patches or placebo patches for 2 weeks, followed by 1 week on placebo in all patients. We hypothesized that subjects who received placebo patches would experience greater withdrawal symptoms upon cessation, show an increase in depressive symptoms, and be more likely to fail at cessation than subjects who received active patch therapy.

\section{METHOD}

\section{Subjects}

Subjects were 38 (20 male, 18 female) unmedicated, outpatient, cigarette smokers with major depression without psychotic features as specified in the DSM-III-R 
(American Psychiatric Association and American Psychiatric Association, Work Group to Revise DSM-III 1987), who obtained a score of $\geq 14$ on the Hamilton Rating Scale for Depression (HRSD, 17 item) (Hamilton 1960). Subjects were required to have smoked at least one pack of cigarettes per day for at least a year, and smoking was biochemically confirmed using an expired air carbon monoxide level of $15 \mathrm{ppm}$ or greater at intake evaluation. Additional inclusion criteria were age 18 years or older, motivation to quit smoking and willingness to comply with study demands. Exclusionary criteria included use of any psychotropic medication for at least 2 weeks prior to initiation of the protocol, symptoms of psychosis, signs of suicidality, significant medical history that might be affected by nicotine, serious dermatological disease, or history of alcohol or drug abuse 1 year prior to study. In addition women were excluded if they were pregnant, lactating, or of childbearing potential (not using medically acceptable form of birth control).

Subjects were recruited through advertisements in print media over a period of 4 years, and those who responded were first screened briefly by telephone to assess the inclusion/exclusion criteria. Following telephone screening, subjects met with a physician or a trained research technician who administered the Structured Clinical Interview for Diagnosis (SCID) (Spitzer et al. 1987) and HRSD. In addition, all subjects underwent a complete physical examination, blood tests, and urine test to test for drug use and pregnancy, and electrocardiogram to test for heart problems.

A total of 38 subjects entered the study, and after giving their informed consent they were randomly as- signed to active $(\mathrm{N}=18)$ and placebo $(\mathrm{N}=20)$ groups. Subjects were dropped from the study if they either resumed smoking following the target quit date (TQD) (defined as smoking 3 cigarettes or more per self-report) or if their clinical depressive symptoms worsened substantially. All subjects, both completers and non-completers, were offered treatment for depression after their study participation. Table 1 shows patient baseline demographic characteristics, psychiatric and smoking history, and mood ratings, by treatment group. Statistical tests comparing the two groups revealed no significant baseline differences, with the exception that subjects assigned to the active patch group had significantly higher scores on the HRSD $(\mathrm{t}(36)=2.3, p<.05)$.

\section{Transdermal Nicotine}

Hoechst Marion Roussel Inc. (Kansas City, MO) supplied the transdermal patches used in this study (both active and placebo). The active nicotine patches delivered $21 \mathrm{mg}$ of nicotine over $24 \mathrm{hr}$. The placebo patches contained $22 \mathrm{mg}$ of nicotine with a barrier to prevent absorption. Subjects were instructed to apply a new patch each morning and to rotate patch placement sites to prevent skin irritation.

\section{Measures}

Mood was assessed in three different ways to capture different aspects of depressive symptoms. First, observer ratings were collected weekly by trained research staff using the HRSD. Second, subjective ratings of depression were collected three times a week with the Beck Depression In-

Table 1. Demographics, Smoking and Psychiatric History, and Baseline Mood Ratings by Treatment Group

\begin{tabular}{lccc}
\hline & \multicolumn{3}{c}{ Treatment group } \\
\cline { 2 - 4 } & Placebo & Active & Total \\
& $\mathbf{( N = \mathbf { 2 0 } )}$ & $\mathbf{( N = 1 8 )}$ & $\mathbf{( N = 3 8 )}$ \\
\hline Age & $45.5(8.2)$ & $47.1(11.1)$ & $46.2(9.6)$ \\
Gender (\% males) & 60.0 & 44.4 & 52.6 \\
Marital status (\% married) & 25.0 & 38.9 & 31.6 \\
Ethnicity (\% Caucasian) & 85.0 & 83.3 & 84.2 \\
Age started smoking & $16.2(3.8)$ & $18.1(6.6)$ & $17.1(5.3)$ \\
Years smoked & $27.7(8.7)$ & $28.2(13.2)$ & $27.9(11.0)$ \\
Packs per day & $1.4(.5)$ & $1.5(.4)$ & $1.4(.4)$ \\
Serious attempts to quit & $3.6(4.6)$ & $6.9(14.4)$ & $4.9(9.7)$ \\
Fagerstrom Test for Nicotine Dependence & $7.2(1.7)$ & $7.1(1.4)$ & $7.1(1.6)$ \\
Age at first depressive episode & $31.4(14.8)$ & $34.2(12.7)$ & $32.7(13.7)$ \\
Number of depressive episodes & $2.8(3.9)$ & $2.1(.83)$ & $2.5(2.9)$ \\
Number of psychiatric hospitalizations & $.30(.66)$ & $.35(.61)$ & $.32(.63)$ \\
Hamilton Rating Scale for Depression* & $17.1(3.0)$ & $19.2(2.8)$ & $18.1(3.1)$ \\
Beck Depression Inventory & $21.0(6.7)$ & $20.7(5.7)$ & $20.9(6.2)$ \\
Profile of Mood States: Total Mood Disturbance & $80.5(34.6)$ & $77.1(24.3)$ & $78.9(29.8)$ \\
\hline
\end{tabular}

Values are means and standard deviations unless otherwise stated.

${ }^{*}$ Mean scores significantly different $(p<.05)$ between active and placebo groups on $t$-test. 
ventory (BDI) (Beck et al. 1987). And third, more acute and transitory mood states were assessed three times a week with the Profile of Mood States (POMS) questionnaire (McNair et al. 1971). Withdrawal symptoms were assessed daily with two different self-report questionnaires. First, they were assessed using the Hughes-Hatsukami Withdrawal Questionnaire (HHWQ), a valid and reliable measure of tobacco withdrawal (Hughes and Hatsukami 1986). Eight symptoms based on the DSM-III-R criteria are rated with reference to the past $24 \mathrm{hr}$ using a 5-point scale $(0-4)$ : desire to smoke; anger irritability, frustration; anxiety, nervousness; difficulty concentrating; impatience, restlessness; hunger; awakening at night; and depression. Secondly, withdrawal symptoms were assessed with an 18 item Nicotine Symptom Checklist where subjects rated the severity of the following physical and mental symptoms on a 5 point scale (0-4): dry mouth, salivation, tiredness, yawning, muscle aches, joint aches, anxiety, elevated mood, increased energy, poor concentration, visual disturbances, nausea, vomiting, diarrhea, dizziness, increased urination, skin reaction, sweating. Subjects also reported daily whether over the past $24 \mathrm{hr}$ they had smoked any cigarettes (even a puff), used any forms of nicotine other than cigarettes, and whether they had consumed any alcohol. Subjects were instructed to complete self-reports at night, preferably at the same time of the day. In addition, subjects completed the Fagerstrom Test for Nicotine Dependence (FTND) at baseline (Heatherton et al. 1991).

\section{Procedure and Design}

The study had three phases: an acute phase which lasted for the first 29 days; a 10 week maintenance phase where subjects were offered standard nicotine patches as well as treatment for their depression; and a follow-up phase for up to a year, with evaluation of mood and smoking status at 3, 6, and 12 months. The data reported here are from the 29-day acute phase.

After subjects had been screened at intake to assess if they met inclusion/exclusion criteria, subjects were started in the acute phase during which time they did not receive antidepressant or other psychotropic medication. All subjects were started in the study on the same day of the week (Tuesday), and during the first 7 days (baseline) all subjects were allowed to smoke their usual amount. On day 8, all subjects were instructed to stop smoking (TQD) and were started, under double blind conditions, on transdermal patches (nicotine or placebo) for a period of 14 days. After the 2-week treatment period (day 22) all subjects received placebo patches for 7 days. During the 29 days, subjects met weekly (on Thursdays) in a counseling/support group led by a trained research assistant, who also had telephone contact with subjects once a week to offer support and assess progress. Subjects met weekly (on Tuesdays) with medical staff where ratings were done
(HRSD), blood samples collected, CO levels measured, and self-reports collected.

The design of the study was therefore a mixed design with treatment group as a between subjects factor (active and placebo) and study phase (baseline, treatment, and placebo) as within a subjects factor. We hypothesized that subjects in the placebo group would be more likely to fail at abstinence, that they would show more symptoms of withdrawal during the treatment phase (as measured by HHWQ and NSC), and that they would show an increase in depressive symptoms following cessation as measured by the three different measures of mood (HRSD, BDI, POMS).

\section{RESULTS}

\section{Abstinence}

Of the 38 subjects who started the study, 13 subjects resumed smoking after TQD and were therefore dropped from the study. One additional subject in the placebo group was dropped from the study because of both depressive symptoms and smoking. Consistent with the literature on the effectiveness of nicotine replacement treatment in smoking cessation trials (Hughes et al. 1999) significantly more subjects resumed smoking by day 29 in the placebo group (50\%) than in the active group (22\%) [ $\chi^{2}=3.1, \mathrm{df}=1, p<.05$ (one sided)].

The rate of abstinence was analyzed further using survival analysis. Figure 1 shows the proportion of subjects not smoking during the 3 weeks following cessation of smoking. As the figure shows, the subjects who resumed smoking did so during the first 10 days after quitting. The proportion of non-smokers was higher in the active than placebo group, but the difference did not reach statistical significance using the Wilcoxon summed ranks test $(\mathrm{S}=2.3, \mathrm{df}=1$, n.s. $)$.

To understand why some patients resumed smoking we examined the data in two ways. First, we examined whether completers and non-completers had different mood ratings at baseline and whether they had different smoking histories. As shown in Table 2, completers had significantly higher ratings at baseline on both the HRSD and the POMS-Total Mood Disturbance scale (POMS-TMD) compared with non-completers; smoking histories were similar across the 2 groups. Second, we examined whether completers and non-completers had experienced differential changes in mood or withdrawal ratings from baseline to treatment. The effects of status (completer vs. non-completer) and study phase were examined using mixed model ANOVA, with study phase as a within factor and status as a between factor. This analysis was somewhat complicated by the fact that we did not have data from the treatment phase for all of the non-completers (since they had been dropped from the study before ratings had been col- 


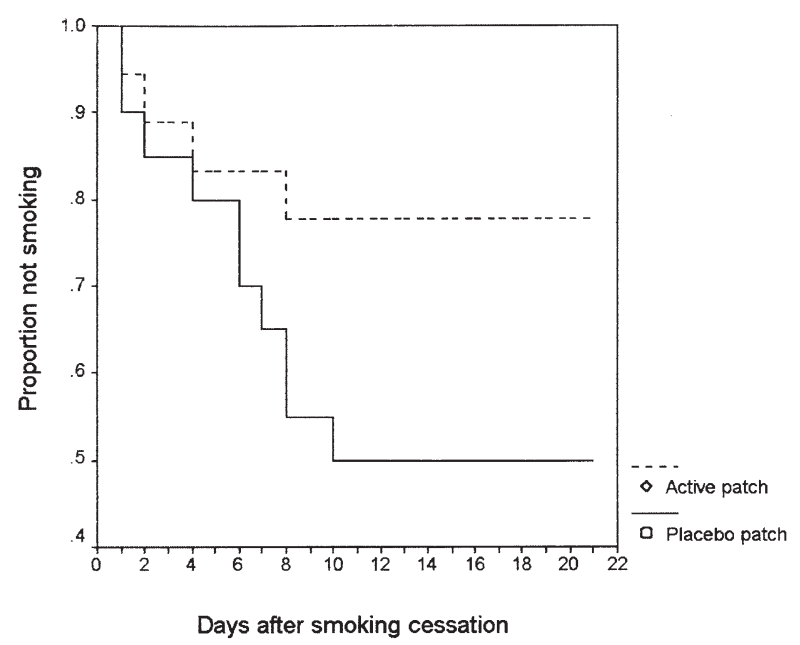

Figure 1. Proportion of subjects not smoking by treatment group $(\mathrm{S}=2.3 \mathrm{df}=1$, n.s. $)$.

lected). But using the available data, we found no significant interaction of study phase and status for mood ratings (HRSD, BDI, POMS), while there was a significant interaction on 2 (out of 18) items from the Nicotine Symptom Checklist and 5 (out of 8) items on the Hughes-Hatsukami Withdrawal Questionnaire (see Table 3). These significant interactions were followed up by calculating paired $t$-tests separately for completers and non-completers. On 6 of the 7 items completers showed no significant change from baseline to treatment phase, and on 1 item (desire to smoke) they showed a significant improvement. In contrast, among non-completers withdrawal ratings were significantly higher during treatment than during baseline on 6 of the 7 items, but did not reach significance on the "Dizziness" item on the NSC ( $p=.06)$.

\section{Withdrawal Ratings}

As shown in Figure 2, the placebo group had somewhat higher overall withdrawal ratings during the treatment phase on both the NSC and the HHWQ, as we had pre- dicted. However, when the effects of study phase and experimental group were tested (using Mixed Model ANOVA, with Huyn-Feldt adjustment to degrees of freedom), we found no significant interaction between study phase and experimental group or significant main effects. Analysis of individual items on the NSC and the HHWQ also failed to show any interactions of study phase and experimental group.

\section{Mood Ratings}

As shown in Figure 3 average mood ratings among completers tended to decrease over the course of the study. We had predicted that the active patches would have antidepressant properties, resulting in an interaction between experimental group and study phase. Statistical analyses using Mixed Model ANOVA with Huyn-Feldt corrections to degrees of freedom revealed a significant interaction for the POMS-TMD scores, but not the HRSD or the BDI. We explored the interaction on the POMS-TMD further in two ways. First, we examined group differences during baseline, treatment, and placebo phases using t-tests. No significant differences were found between the groups. Second, we examined the effect of study phase separately for the two treatment groups. This analysis revealed that there was a significant effect of study phase within the placebo group with scores getting lower over time, while there was no effect of study phase within the active group. Scores on the subscales of the POMS were also analyzed and the pattern of results did not support our hypothesis. For the sake of brevity the results on the POMS subscales are not presented here. The main effects of study phase and experimental group were analyzed for HRSD and BDI scores. The analyses revealed a significant main effect of study phase for HRSD scores, while study phase had no effects on BDI scores. Experimental group had no effect on either HRSD or BDI scores.

The effects of treatment and study phase on mood ratings were also examined with last observation carried forward, which produced largely similar findings.

Table 2. Baseline Mood Ratings, Nicotine Dependence, and Smoking among Study Completers and Non-Completers

\begin{tabular}{|c|c|c|c|}
\hline & $\begin{array}{l}\text { Non-completers } \\
\quad(\mathbf{N}=14)\end{array}$ & $\begin{array}{l}\text { Completers } \\
(\mathrm{N}=24)\end{array}$ & $\begin{array}{c}\text { Total } \\
(\mathrm{N}=38)\end{array}$ \\
\hline Hamilton Rating Scale for Depression* & $16.8(2.1)$ & $18.8(3.3)$ & $18.1(3.1)$ \\
\hline Beck Depression Inventory & $19.4(6.7)$ & $21.7(5.8)$ & $20.9(6.2)$ \\
\hline Profile of Mood States: Total Mood Disturbance* & $61.0(18.8)$ & $89.3(30.3)$ & $78.9(29.8)$ \\
\hline Fagerstrom Test for Nicotine Dependence & $7.4(1.4)$ & $6.9(1.7)$ & $7.1(1.6)$ \\
\hline Years smoked & $26.8(8.1)$ & $28.5(12.3)$ & $27.9(11.0)$ \\
\hline Packs per day & $1.4(0.4)$ & $1.4(0.3)$ & $1.4(0.4)$ \\
\hline Age started smoking & $17.5(4.7)$ & $17.3(5.9)$ & $17.4(5.4)$ \\
\hline
\end{tabular}

${ }^{*}$ Mean scores are significantly different $(p<.05)$ between completers and non-completers on $t$-test. 
Table 3. Withdrawl Ratings for Study Non-Completers and Completers During Baseline and Treatment Phases

\begin{tabular}{|c|c|c|c|c|}
\hline & \multicolumn{2}{|c|}{ Non-Completers } & \multicolumn{2}{|c|}{ Completers } \\
\hline & Baseline & Treatment & Baseline & Treatment \\
\hline Nicotine Symptom Checklist & \multicolumn{2}{|c|}{$(\mathrm{N}=8)$} & \multicolumn{2}{|c|}{$(\mathrm{N}=18)$} \\
\hline Poor Concentration $^{a}$ & $1.4(0.9)$ & $2.3(1.0)^{*}$ & $1.9(1.0)$ & $1.9(1.2)$ \\
\hline Dizziness $^{b}$ & $0.2(0.4)$ & $0.7(0.7)$ & $0.2(0.4)$ & $0.2(0.5)$ \\
\hline Hughes-Hatsukami Withdrawl Questionnaire & \multicolumn{2}{|c|}{$(\mathrm{N}=9)$} & \multicolumn{2}{|c|}{$(\mathrm{N}=23)$} \\
\hline Desire to smoke ${ }^{c}$ & $2.6(0.8)$ & $3.4(0.6)^{*}$ & $3.5(0.5)$ & $2.9(0.7)^{*}$ \\
\hline Anger, Irritability, Frustration ${ }^{d}$ & $1.1(0.8)$ & $2.2(1.0)^{*}$ & $2.0(1.0)$ & $2.3(0.9)$ \\
\hline Anxiety, Nervousness ${ }^{e}$ & $1.6(1.0)$ & $2.4(1.0)^{*}$ & $2.2(1.0)$ & $2.4(0.9)$ \\
\hline Difficulty Concentrating $f$ & $1.4(0.9)$ & $2.4(1.2)^{*}$ & $2.4(1.0)$ & $2.2(1.1)$ \\
\hline Impatience, Restlessness ${ }^{g}$ & $1.5(0.9)$ & $2.5(1.0)^{*}$ & $2.3(1.0)$ & $2.4(0.9)$ \\
\hline
\end{tabular}

Values in table are means and standard deviations (in parentheses)

*Mean scores within groups for baseline and treatment significantly different on paired $t$-test $(p<.05)$.

Significant interaction of study phase and status: ${ }^{a} \mathrm{~F}_{1,24}=8.36, p<.01 ;{ }^{b} \mathrm{~F}_{1,24}=5.6, p<.05 ;{ }^{c} \mathrm{~F}_{1,30}=16.1, p<$ $.001 ;{ }^{d} \mathrm{~F}_{1,30}=4.3, p<.05 ;{ }^{e} \mathrm{~F}_{1,30}=4.3, p<.05 ;{ }^{f} \mathrm{~F}_{1,30}=9.2, p<.01 ;{ }^{8} \mathrm{~F}_{1,30}=5.6, p<.05$.

\section{DISCUSSION}

This study is the first prospective, randomized clinical trial to report on the effects of smoking cessation on currently depressed patients. The goal was to examine the effects of transdermal nicotine patches on mood in subjects with MDD undergoing smoking cessation.

The results show that among those who remained abstinent over the 29 day study period $(\mathrm{N}=24) \operatorname{mood}$ ratings improved slightly rather than worsening. As an example, HRSD ratings declined from a mean of 18.8 during baseline, to a mean of 16.4 during the treatment phase and to a mean of 16.2 during the placebo phase. POMS-TMD scores declined by $18 \%$ from the baseline to the placebo phase (89.3 vs. 73.5 ). The results did not support our hy- pothesis that the group receiving nicotine patches would experience less depressive symptoms following cessation. In addition, the results in the entire group $(\mathrm{N}=38)$ produced similar findings, with no evidence for either increased depression following cessation in the placebo group or antidepressant effects on mood in the nicotine group. Nevertheless, one subject had a substantial increase in depressive symptoms and he was in the group of 20 subjects who received placebo patches. This subject (a 35-year-old male who had smoked for 22 years and had a history of 2 psychiatric hospitalizations) started the study with a HRSD rating of 22, which dropped to 12 and 14 on days 8 and 15 of the study. On day 22 of the study (day 15 on placebo patch) the HRSD rating had increased to 31, and the subject was subsequently dropped from the
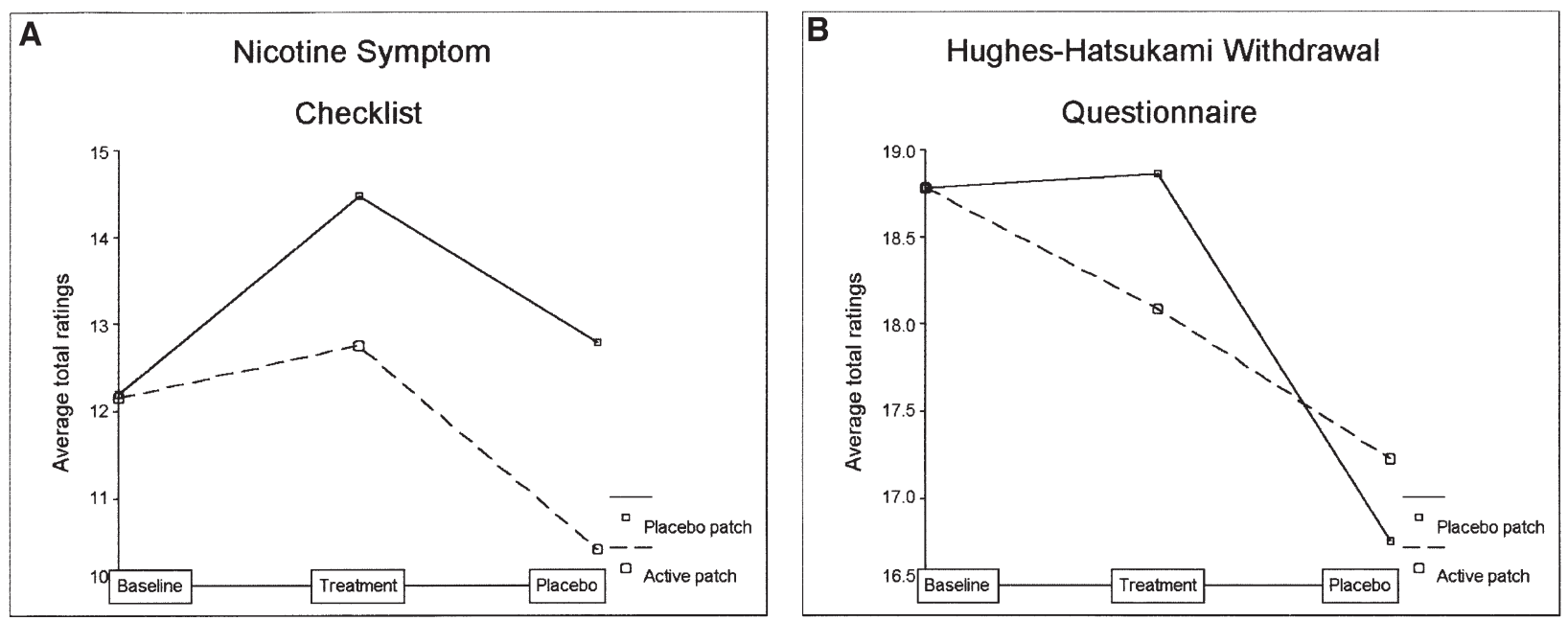

Figure 2. Average total withdrawal ratings on the Nicotine Symptom Checklist and Hughes-Hatsukami Withdrawal Questionnaire by treatment group and study phase in completers. (A) Main effects of study phase $[\mathrm{F}(1.4,22.7)=1.8]$, experimental group $[\mathrm{F}(1,16)=2]$, and interaction $[\mathrm{F}(1.4,22.7)=6]$ nonsignificant. (B) Main effects of study phase $[\mathrm{F}(1.5,32.4)=$ $1.4]$, experimental group $[\mathrm{F}(1,21)=.002]$, and interaction $[\mathrm{F}(1.5,32.4)=.1]$ non-significant. 

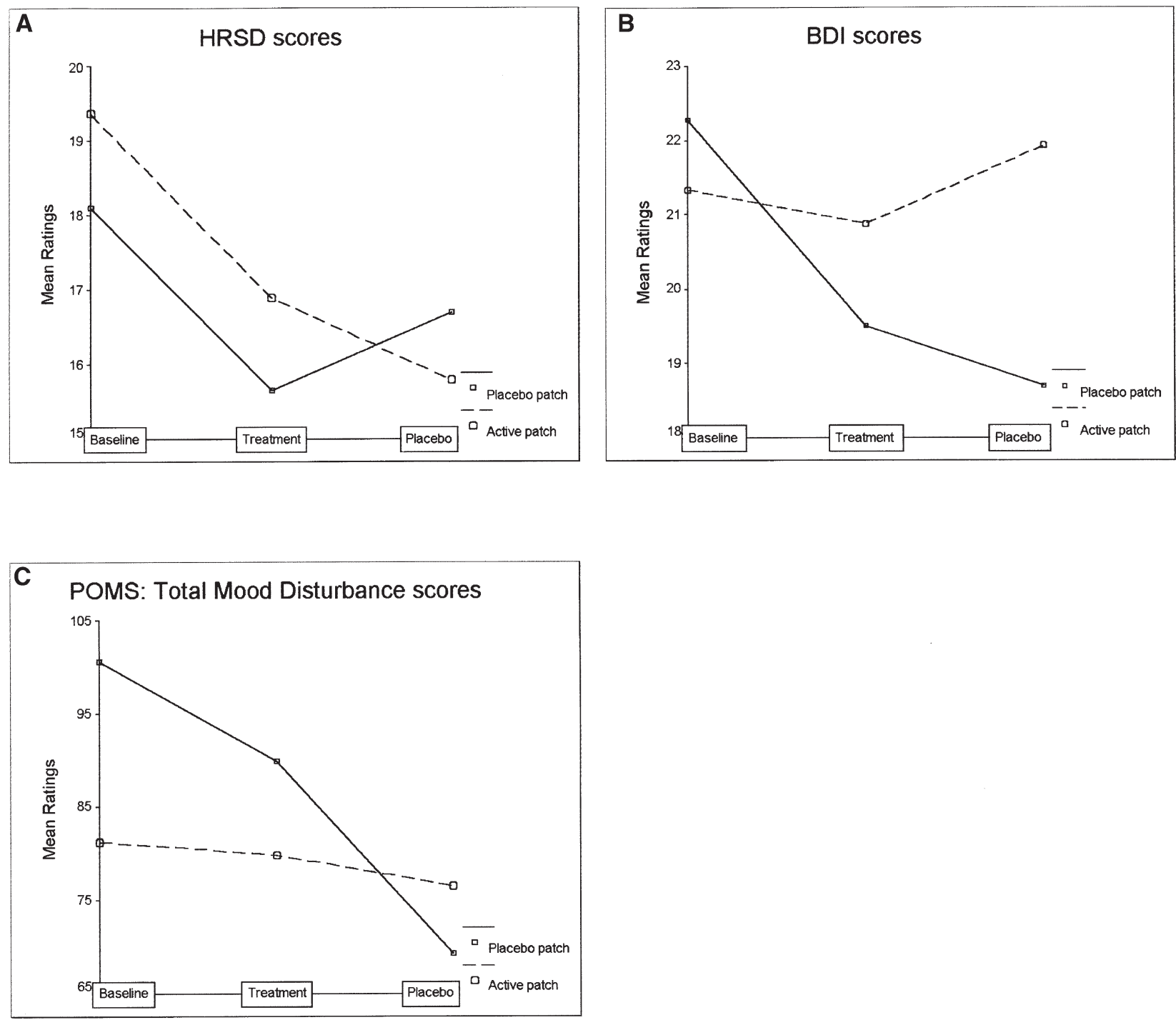

Figure 3. Mean mood ratings for completers during baseline, treatment and placebo phases of study by treatment group. (A) Interaction non-significant $[\mathrm{F}(1.7,36.3)=1.2]$. Significant main effect of study phase $[\mathrm{F}(1.7,36.3)=6.1, p=.01]$. No effect of experimental group $[\mathrm{F}(1,22)=.1]$. (B) Interaction non-significant $[\mathrm{F}(1.9,41.1)=2.4]$. No effect of study phase $[\mathrm{F}(1.9,41.1)=$ $1.8]$ and experimental group $[\mathrm{F}(1,22)=.2]$. (C) Significant interaction $[\mathrm{F}(1.6,35.2)=5.8, p<.05]$. No significant group differences at baseline, treatment, placebo phases. Significant effect of study phase within placebo group $[\mathrm{F}(1.5,9)=7.9, p<.01]$, but not within active group.

study. None of the 18 patients started on active patches became substantially more depressed.

The dropout rate was greater in the group receiving placebo $(50 \%)$ than nicotine $(22 \%)$ patches. This differential dropout rate may have masked the effects of smoking cessation on depressive symptoms, but our analyses comparing completers and non-completers failed to show any evidence in that direction. Non-completers did not have higher baseline mood ratings than completers, nor did their mood ratings worsen more than among completers following cessation.

We acknowledge that the study may be underpowered, but it was impossible to run more subjects be- cause of time and money constraints and because of difficulty recruiting depressed patients who were willing to stop smoking in a demanding clinical trial. The observed power (at $\alpha=.05$ ) for tests of the main effects for study phase (baseline, treatment, and placebo) for HRSD, BDI, and POMS-TMD was .81, .34, and .97 respectively. For the interaction of treatment (nicotine vs. placebo) and study phase it was $.22, .44$, and .78 for the same variables at the same $\alpha$-level. As these values show some tests were under-powered, but other tests had adequate power.

We justify the use of a one-tailed test in testing rates of abstinence between the experimental groups be- 
cause we had an a priori hypothesis based on numerous studies of the clinical effectiveness of nicotine replacement therapy in smoking cessation. Furthermore, the use of one-tailed tests has been justified in the literature by Overall (1990) for clinical pharmacological trials where a hypothesis predicts a better response over placebo.

Our findings therefore offer little support for two hypotheses: a) that smoking cessation increases the severity of depression in unmedicated depressed patients, and $b$ ) the administration of nicotine may ameliorate depressive symptoms in unmedicated depressed patients who are quitting. Previous findings have shown that euthymic patients with a history of MDD are at risk of developing MDD following smoking cessation (Bock et al. 1996; Borrelli et al. 1996b; Covey et al. 1997), and we therefore expected that the depressed patients in our study would experience an increase in depression after cessation. It is unclear why our patients showed an overall improvement and smoking cessation was not related to a decline in mood, but several factors may have contributed to these results. First, all subjects in our study received considerable professional attention during the study period. They met weekly with clinical research staff, attended weekly support groups and were contacted once a week by telephone by the group leader. These factors may have been responsible for some improvement in depressive symptoms among our subjects. Second, the patients may have experienced a sense of pride and achievement from being able to abstain from smoking. Third, it is possible that the onset of depression following smoking cessation may be delayed and that the 3-week follow-up period used in this study may not have been long enough. Only two of the studies of depression following smoking cessation report how long after quit date the onset of depression occurred. The three cases reported by Bock et al. (1996) all developed depressive symptoms within 3 weeks after cessation, but the five cases reported by Patten et al. (1999) developed depression 4 to 45 weeks after cessation. Fourth, it should be pointed out that in previous studies, only a subset of patients with a history of MDD became symptomatic following cessation. Only two out of the three studies report the rates among euthymic patients with a history of MDD who became symptomatic following cessation, which were $21 \%$ (7 out of 34 patients) (Covey et al. 1997) and 14\% (5 out of 69 patients) (Borrelli et al. 1996b). In comparison $3 \%$ (1 patient out of 38 ) in our study experienced a substantial increase in depression. It is, therefore, possible that only a subset of smokers with MDD are vulnerable to the depressant effects of smoking cessation. Finally, it is possible that smoking cessation may trigger an episode of depression in euthymic patients who are at risk, but not affect the severity of an ongoing depressive episode. This study used a sample of patients who were depressed at the time of study, whereas previous studies (Bock et al. 1996; Borrelli et al. 1996b; Covey et al. 1997) all excluded patients who were depressed at the time of study.

The finding that nicotine patches had no clear antidepressant effects was unexpected since previous studies have shown that nicotine may have antidepressant properties (Pomerleau and Pomerleau 1992; Salin-Pascual et al. 1995; Salin-Pascual et al. 1996; Salin-Pascual and Drucker-Colín 1998). Further studies are needed to address this discrepancy.

Taken together, the modest improvement we observed in mood following cessation in both groups of subjects, and that nicotine patches had no clear antidepressant effects, may offer some insight into the causal relationship between depression and smoking. If smoking cessation causes depression, we should have observed a clear decline in mood upon cessation, which we did not. And if the self-medication model is true (i.e. that depressed people use nicotine to enhance their mood), we should have observed better mood upon cessation among those receiving nicotine patches, which we did not. Our results are therefore consistent with findings that suggest that smoking and depression may not be causally related (Fergusson et al. 1996; Kendler et al. 1993)

Our data provided trend support for the hypothesis that smoking cessation would increase withdrawal symptoms and that nicotine patches would ameliorate these effects. These effects, however, did not reach statistical significance. Nevertheless, rates of relapse were significantly higher in the placebo group $(50 \%)$ than the nicotine group (22\%). Subjects who resumed smoking had more severe withdrawal symptoms prior to relapse than those who remained abstinent. They reported poorer concentration, more desire to smoke, more anger, irritability and frustration, more anxiety and nervousness, and more impatience and restlessness during the first days of smoking cessation than those who remained abstinent for the study period.

Since the subjects in this study were not randomly sampled from the population of depressed smokers we cannot generalize much from these findings to other depressed smokers. Those who decided to participate in our study may have been more motivated to quit than the average depressed smoker. However, our findings suggest that if depressed patients are well motivated to quit and receive some support during the first weeks of cessation, they may not be at risk for increased depression, and they may actually show improvement in their mood. Depressed patients who quit on their own or with less behavioral support than was offered in this study, may have more difficulty in quitting with respect to depressive symptoms. Our findings also suggest that depressed patients are more likely to abstain from smoking during the first weeks of cessation if they use nicotine patch therapy. 


\section{ACKNOWLEDGMENTS}

Supported in part by a grant form NIH to UCSD MHCRC MH30914, and a grant from Tobacco Related Disease Research Program \#4RT-0301 (JCG).

\section{REFERENCES}

American Psychiatric Association, Work Group to Revise DSM-III (1987): Diagnostic and statistical manual of mental disorders: DSM-III-R. Washington, DC, American Psychiatric Association

Anda RF, Williamson DF, Escobedo LG, Mast EE, Giovino GA, Remington PL (1990): Depression and the dynamics of smoking. A national perspective. JAMA 264:1541-1545

Beck A, Ward C, Mendelson M, Mock J, Erbaugh J (1987): Manual for the Beck Depression Inventory. New York, Harcourt Brace Jovanovich

Bock BC, Goldstein MG, Marcus BH (1996): Depression following smoking cessation in women. J Subst Abuse 8:137-144

Borrelli B, Bock B, King T, Pinto B, Marcus BH (1996a): The impact of depression on smoking cessation in women. Am J Prev Med 12:378-387

Borrelli B, Niaura R, Keuthen NJ, Goldstein MG, DePue JD, Murphy C, Abrams DB (1996b): Development of major depressive disorder during smoking-cessation treatment. J Clin Psychiatry 57:534-538

Breslau N (1995): Psychiatric comorbidity of smoking and nicotine dependence. Behav Genet 25:95-101

Breslau N, Kilbey M, Andreski P (1991): Nicotine dependence, major depression, and anxiety in young adults. Arch Gen Psychiatry 48:1069-1074

Breslau N, Peterson EL, Schultz LR, Chilcoat HD, Andreski P (1998): Major depression and stages of smoking. A longitudinal investigation. Arch Gen Psychiatry 55:161-166

Covey LS, Glassman AH, Stetner F (1997): Major depression following smoking cessation. Am J Psychiatry 154:263-265

Covey LS, Glassman AH, Stetner F, Becker J (1993): Effect of history of alcoholism or major depression on smoking cessation. AM J Psychiatry 150:1546-1547

Fergusson DM, Lynskey MT, Horwood LJ (1996): Comorbidity between depressive disorders and nicotine dependence in a cohort of 16-year-olds. Arch Gen Psychiatry 53:1043-1047

Fowler JS, Volkow ND, Wang GJ, Pappas N, Logan J, MacGregor R, Alexoff D, Shea C, Schlyer D, Wolf AP, Warner D, Zezulkova I, Cilento R (1996): Inhibition of monoamine oxidase B in the brains of smokers. Nature 379:733-736

Ginsberg D, Hall SM, Reus VI, Munoz RF (1995): Mood and depression in smoking cessation. Exp Clin Psychopharmacol 3:389-395

Glassman AH, Covey LS, Dalack GW, Stetner F, Rivelli SK, Fleiss J, Cooper TB (1993): Smoking cessation, clonidine, and vulnerability to nicotine among dependent smokers. Clin Pharmacol Ther 54:670-679

Glassman AH, Helzer JE, Covey LS, Cottler LB, Stetner F, Tipp JE, Johnson J (1990): Smoking, smoking cessation, and major depression. JAMA 264:1546-1549
Glassman AH, Stetner F, Walsh BT, Raizman PS, Fleiss JL, Cooper TB, Covey LS (1988): Heavy smokers, smoking cessation, and clonidine. Results of a double-blind, randomized trial. JAMA 259:2863-2866

Hamilton M (1960): A Rating Scale for Depression. J Neurol Neurosurg Psychiatry 23:56-61

Heatherton TF, Kozlowski LT, Frecker RC, Fagerström KO (1991): The Fagerström Test for Nicotine Dependence: a revision of the Fagerström Tolerance Questionnaire. Br J Addict 86:1119-1127

Hughes JR, Goldstein MG, Hurt RD, Shiffman S (1999): Recent advances in the pharmacotherapy of smoking. JAMA 281:72-76

Hughes JR, Hatsukami D (1986): Signs and symptoms of tobacco withdrawal. Arch Gen Psychiatry 43:289-294

Hughes JR, Hatsukami DK, Mitchell JE, Dahlgren LA (1986): Prevalence of smoking among psychiatric outpatients. Am J Psychiatry 143:993-997

Kendler KS, Neale MC, MacLean CJ, Heath AC, Eaves LJ, Kessler RC (1993): Smoking and major depression. A causal analysis. Arch Gen Psychiatry 50:36-43

Lee DJ, Markides KS (1991): Health behaviors, risk factors, and health indicators associated with cigarette use in Mexican Americans: Results from Hispanic HANES. Am J Public Health 81:859-864

Lerman C, Caporaso N, Main D, Audrain J, Boyd NR, Bowman ED, Shields PG (1998): Depression and self-medication with nicotine: The modifying influence of the dopamine D4 receptor gene. Health Psychol 17:56-62

McNair D, Lorr M, Droppleman LF (1971): Manual for the Profile of Mood States. San Diego, CA EDITS Overall JE (1990): Tests of one-sided versus two-sided hypotheses in placebo-controlled clinical trials. Neuropsychopharmacology 3:233-235

Overall JE (1990): Tests of one-sided versus two-sided hypotheses in placebo-controlled clinical trials. Neuropsychopharmacology 3:233-235

Patten CA, Rummans TA, Croghan IT, Hurt RD, Hays JT (1999): Development of depression during placebo-controlled trials of bupropion for smoking cessation: case reports. J Clin Psychiatry 60:436-441

Pomerleau CS, Pomerleau OF (1992): Euphoriant effects of nicotine in smokers. Psychopharmacology 108:460-465

Salin-Pascual RJ, de la Fuente JR, Galicia-Polo L, DruckerColin R (1995): Effects of transdermal nicotine on mood and sleep in nonsmoking major depressed patients. Psychopharmacology 121:476-479

Salin-Pascual RJ, Drucker-Colín R (1998): A novel effect of nicotine on mood and sleep in major depression. Neuroreport 9:57-60

Salin-Pascual RJ, Rosas M, Jimenez-Genchi A, Rivera-Meza BL, Delgado-Parra V (1996): Antidepressant effect of transdermal nicotine patches in nonsmoking patients with major depression. J Clin Psychiatry 57:387-389

Salive ME, Blazer DG (1993): Depression and smoking cessation in older adults: A longitudinal study. J Am Geriatr Soc 41:1313-1316

Spitzer RL, Williams JBW, Gibbon M (1987): Structured Clinical Interview for DSM-III-R (SCID). New York, Biometric Research, New York State Psychiatric Institute 